Mean age 66.5 years

Percent of female $50.47 \%$

Unplanned admissions 82.28\%

Over the following 365 days, of the 858 :

Number of patients that died 223 (25.99\%)

Number that died in CUH 51 (5.94\%)

Median number if $\mathrm{A}$ and $\mathrm{E}$ attendance 2

The median bed days in the census year 40

The median number of admissions in the census year 3

Higher rates in geriatrics and oncology as expected. Data is being used to engage clinicians in End of LIfe Care and point out that hospitals are in a good position to provide advance care and EOLC planning

\section{AN AUDIT OF DOCUMENTATION OF CARDIOPULMONARY RESUSCITATION (CPR) DECISION MAKING AND COMMUNICATION ON ADMISSION TO A HOSPICE INPATIENT UNIT}

Hazel Gilkes. Marie Curie Hospice Bradford, UK

\subsection{6/bmjspcare-2018-ASPabstracts.106}

Background CPR decisions are a significant part of advanced care planning. Clear communication with patients and those important to them, and documentation of decision making, is essential for good care and, following court cases, is now also a legal requirement.

Purpose The audit aim was to assess documentation of CPR decisions and communication on admission to Marie Curie Hospice Bradford and review CPR documentation on the patient's electronic palliative care co-ordination system (EPaCCS).

Methods Standards were set using Resuscitation Council (UK) and GMC guidance and the hospice's CPR policy. All admissions between 1 st-31st January 2017 were audited and results compared to an initial audit in 2016. The 2017 audit was extended to assess CPR decision making documentation on EPaCCS.

Results 38 admissions were audited. All had a CPR decision documented of which 32 were DNACPR. 22 were discussed with patients, 9 with relatives and 8 were not discussed. In 2016 of 37 admissions audited, all had a CPR decision. Only 13 DNACPR decisions were discussed with patients.

On EPaCCS, of the 10 DNACPR decisions not discussed at admission, 6 were discussed previously with patients and 2 were not discussed.

Conclusions and recommendations In both audits 2 audit standards were met:

- All patients should have a decision about CPR at the time of admission.

- CPR decisions and rationale should be clearly documented.

2 standards were not met:

- CPR should be discussed with all patients.

- CPR should be discussed with relatives/those important to the patient.

As the hospice moves to electronic patient records documentation will be modified. In addition to general advanced care plans, 3 specific sections are to be completed for CPR decision, discussion with patient and discussion with relatives.
The aim is to improve documentation of discussions and continuity with EPaCCS. A re-audit will be completed.

\section{WHAT PREVENTS ADVANCE CARE PLANNING IN HAEMATOLOGY?}

Alice Travers, Graeme Donald. Salford Royal NHS Foundation Trust, University of Manchester

\subsection{6/bmjspcare-2018-ASPabstracts. 107}

Background Despite a clear emphasis in policy on the importance of advance care planning (ACP) for achieving quality end of life care, rates of ACP completion remain low. This is particularly apparent for patients with haematological malignancies and is associated with high rates of hospital death and patients receiving aggressive treatments at the end of life. This impacts on quality of life and family bereavement. Although patients tend to be receptive to ACP discussions they expect healthcare professionals to instigate them. Current evidence exploring barriers and facilitators to ACP from the healthcare professionals' perspective is limited.

Aim To explore what doctors and nurses identify as the barriers and facilitators to ACP for patients with haematological malignancies.

Method One-to-one semi-structured interviews were conducted with a purposively recruited sample of 5 doctors and 5 nurses working in a haematological malignancy setting. Thematic data analysis was conducted using a multi-perspectival Interpretative Phenomenological Analysis (IPA) approach. Demographic questionnaires were also used to capture key participant characteristics and provide a rich contextual description of participants. Findings Six super-ordinate themes were identified: the problem with haematology; haematology team culture; approaching the patient; balancing hope and reality; managing patient psychology; opportunities for ACP; and staff awareness and training; each with associated subthemes.

Conclusion Though general claims are cautious, the findings of the study provide indications for future research exploring this phenomenon from the patient's perspective, as well as introducing prompts to trigger early ACP discussions despite prognostic uncertainty. It also poses potential clinical implications to improve holistic patient-centred shared decision-making by: addressing the hierarchical structure of haematology; inter-professional education and ACP awareness promotion; and empowering nurses to initiate ACP.

\section{THE LIVING WELL GROUP: A PUBLIC PARTNERSHIP INITIATIVE TO IMPROVE END OF LIFE CARE IN AN ACUTE HOSPITAL}

Amy Pharaoh, Clive Hunt. Poole Hospital NHS Foundation Trust, Forest Holme Hospice

\subsection{6/bmjspcare-2018-ASPabstracts. 108}

Background Poole Hospital NHS Foundation Trust is participating in Building on the best (Botb) - a national initiative to improve end of life care in acute hospitals. The Ambitions Framework recognises the important role of partnerships between communities and professional services.

A Public Partnership Group was created to support delivery of the projects comprising Botb. 